\title{
Measurement and Fairness
}

\author{
Abigail Z. Jacobs \\ azjacobs@umich.edu \\ University of Michigan
}

\author{
Hanna Wallach \\ hanna@dirichlet.net \\ Microsoft Research
}

\begin{abstract}
We propose measurement modeling from the quantitative social sciences as a framework for understanding fairness in computational systems. Computational systems often involve unobservable theoretical constructs, such as socioeconomic status, teacher effectiveness, and risk of recidivism. Such constructs cannot be measured directly and must instead be inferred from measurements of observable properties (and other unobservable theoretical constructs) thought to be related to them-i.e., operationalized via a measurement model. This process, which necessarily involves making assumptions, introduces the potential for mismatches between the theoretical understanding of the construct purported to be measured and its operationalization. We argue that many of the harms discussed in the literature on fairness in computational systems are direct results of such mismatches. We show how some of these harms could have been anticipated and, in some cases, mitigated if viewed through the lens of measurement modeling. To do this, we contribute fairnessoriented conceptualizations of construct reliability and construct validity that unite traditions from political science, education, and psychology and provide a set of tools for making explicit and testing assumptions about constructs and their operationalizations. We then turn to fairness itself, an essentially contested construct that has different theoretical understandings in different contexts. We argue that this contestedness underlies recent debates about fairness definitions: although these debates appear to be about different operationalizations, they are, in fact, debates about different theoretical understandings of fairness. We show how measurement modeling can provide a framework for getting to the core of these debates.
\end{abstract}

\section{CCS CONCEPTS}

- General and reference $\rightarrow$ Measurement; Validation; Reliability.

\section{KEYWORDS}

measurement, construct validity, construct reliability, fairness

\section{ACM Reference Format:}

Abigail Z. Jacobs and Hanna Wallach. 2021. Measurement and Fairness. In Conference on Fairness, Accountability, and Transparency (FAccT '21), March 3-10, 2021, Virtual Event, Canada. ACM, New York, NY, USA, 11 pages. https://doi.org/10.1145/3442188.3445901

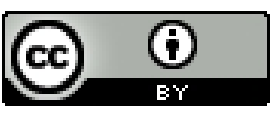

This work is licensed under a Creative Commons Attribution International 4.0 License.

FAccT '21, March 3-10, 2021, Virtual Event, Canada

ACM ISBN 978-1-4503-8309-7/21/03.

https://doi.org/10.1145/3442188.3445901

\section{INTRODUCTION}

Computational systems have long been touted as having the potential to counter societal biases and structural inequalities, yet recent work has demonstrated that they often end up encoding and exacerbating them instead. Indeed, the literature on fairness in computational systems has identified many types of fairness-related harms $[6,16]$, as well as many potential causes, including societal biases reflected in the content of training datasets, too few data points about particular groups of people, and assumptions made throughout the development and deployment lifecycle [e.g., 44]. However, we argue that these discussions have overlooked an important subtlety: computational systems often involve unobservable theoretical constructs-abstractions that describe phenomena of theoretical interest, such as socioeconomic status, teacher effectiveness, and risk of recidivism. Because these constructs cannot be observed, they cannot be measured directly. Instead, they must instead be inferred from measurements of observable properties (and other unobservable theoretical constructs) thought to be related to them-i.e., operationalized via a measurement model. This process-the measurement modeling process- necessarily involves making assumptions, thereby introducing the potential for mismatches between the theoretical understanding of the construct purported to be measured and its operationalization in a measurement model. Indeed, we argue that many of the harms studied in the literature on fairness in computational systems are direct results of such mismatches.

Although it is fundamental to the quantitative social sciences, measurement modeling is largely missing from 1) computer science in general and 2) the discourse around and literature on fairness in computational systems in particular. ${ }^{1}$ As a result, researchers and practitioners are often inclined to conflate constructs and their operationalizations-i.e., to collapse the distinctions between them. But collapsing these distinctions, either colloquially or epistemically, makes it difficult to anticipate, let alone mitigate, any possible mismatches. In other words, collapsing these distinctions elides the space in which fairness-related harms are most often introduced.

In this paper, we propose measurement modeling as a framework for understanding fairness in computational systems. Measurement modeling provides both a language for articulating the distinctions between constructs and their operationalizations and set of toolsnamely construct reliability and construct validity-for surfacing possible mismatches. We argue that these capabilities will enable researchers and practitioners to 1) better anticipate fairness-related harms before deploying computational systems in consequential ways and 2) identify potential causes of fairness-related harms in ways that reveal concrete, actionable avenues for mitigating them.

\footnotetext{
${ }^{1}$ We expect that readers from the quantitative social sciences will already be intimately familiar with measurement modeling. Indeed, some may even wonder why we chose to write this paper. However, we emphasize that many researchers and practitioners outside of these disciplines have not heard of measurement modeling at all, let alone as a framework for understanding fairness in computational systems. We therefore view this paper as contributing an important bridge between disparate disciplines.
} 
We further explain how measurement modeling can contribute to recent debates about fairness definitions. Fairness itself is an unobservable theoretical construct, albeit one that is essentially contested [21, 42]. In other words, fairness has multiple contextdependent, and sometimes even conflicting, theoretical understandings. The contested nature of fairness makes it inherently hard to measure: If there are multiple theoretical understandings of a construct, then it is imperative to articulate which understanding is being operationalized. A failure to do this makes it difficult to meaningfully compare different operationalizations because they may be operationalizing different theoretical understandings. In turn, this makes it difficult to identify mismatches between fairness as it is theoretically understood and any given operationalization.

We argue that although recent debates about fairness definitions have been framed in terms of operationalizations, they are, in fact, debates about different theoretical understandings of fairness-i.e., about the essentially contested nature of fairness as a construct. We show how measurement modeling can get to the core of these debates by providing a language for disentangling debates about different operationalizations of a single theoretical understanding from debates about different, and possibly conflicting, theoretical understandings. This is crucial because debates about different operationalizations of a single theoretical understanding are debates about the measurement modeling process-i.e., the assumptions made when moving from abstractions to mathematics-while debates about different theoretical understandings are debates about values. $^{2}$ We argue that debates about values should be explicitly treated as such instead of being couched indirectly in mathematics, where they are rendered less accessible to the stakeholders that are most likely to be affected by the computational systems in question.

In the next section, we give a brief overview of the measurement modeling process, drawing on well-known examples from the literature on fairness in computational systems. In section 3, we contribute fairness-oriented conceptualizations of construct reliability and construct validity, uniting traditions from political science, education, and psychology. We use the examples introduced in section 2 to illustrate how these conceptualizations can be used to surface mismatches between constructs and their operationalizations. In section 4, we turn to fairness itself, addressing recent debates about fairness definitions. Finally, we conclude with a discussion in section 5 .

\section{MAKING ASSUMPTIONS}

Measurement modeling plays a central role in the quantitative social sciences, where many theories involve unobservable theoretical constructs-i.e., abstractions that describe phenomena of theoretical interest. For example, researchers in psychology and education have long been interested in studying intelligence, while political scientists and sociologists are often concerned with political ideology and socioeconomic status, respectively. Although these constructs do not manifest themselves directly in the world, and therefore cannot be measured directly, they are fundamental to society and thought to be related to a wide range of observable properties.

\footnotetext{
${ }^{2}$ We emphasize that this is not to say that values play no role in the measurement modeling process-quite the contrary, as we explain in the first half of this paper.
}

A measurement model is a statistical model that links unobservable theoretical constructs, operationalized as latent variables, and observable properties-i.e., data about the world [30]. In this section, we give a brief overview of the measurement modeling process, starting with two comparatively simple examples-measuring height and measuring socioeconomic status-before moving on to three well-known examples from the literature on fairness in computational systems. We emphasize that our goal in this section is not to provide comprehensive mathematical details for each of our five examples, but instead to introduce key terminology and, more importantly, to highlight that the measurement modeling process necessarily involves making assumptions that must be made explicit and tested before the resulting measurements are used.

\subsection{Measuring Height}

We start by formalizing the process of measuring the height of a person-a property that is typically thought of as being observable and therefore easy to measure directly. There are many standard tools for measuring height, including rulers, tape measures, and height rods. Indeed, measurements of observable properties like height are sometimes called representational measurements because they are derived by "representing physical objects [such as people and rulers] and their relationships by numbers" [25]. Although the height of a person is not an unobservable theoretical construct, for the purpose of exposition, we refer to the abstraction of height as a construct $\mathcal{H}$ and then operationalize $\mathcal{H}$ as a latent variable $h$.

Despite the conceptual simplicity of height-usually understood to be the length from the bottom of a person's feet to the top of their head when standing erect-measuring it involves making several assumptions, all of which are more or less appropriate in different contexts and can even affect different people in different ways. For example, should a person's hair contribute to their height? What about their shoes? Neither are typically viewed as being an intrinsic part of a person's height, yet both contribute to a person's effective height, which may matter more in ergonomic contexts. Similarly, if a person uses a wheelchair, then their standing height may be less relevant than their sitting height. These assumptions must be made explicit and tested before using any measurements that depend upon them.

In practice, it is not possible to obtain error-free measurements of a person's height, even when using standard tools. For example, when using a ruler, the angle of the ruler, the granularity of the marks, and human error can all result in erroneous measurements. However, if we take $N$ measurements $\left\{\hat{h}_{n}\right\}_{n=1}^{N}$ of a person's height, then provided that the ruler is not statistically biased, the average will converge to the person's "true" height $h$ with probability one as $N \rightarrow \infty$. Specifically, we say that the person's true height-the latent variable $h$-influences the measurements $\left\{\hat{h}_{n}\right\}_{n=1}^{N}$-a set of $N$ observed variables. We can represent this relationship using a graphical model, as shown below. Observed variables are shaded, latent variables are unshaded, and annotated boxes denote replication.

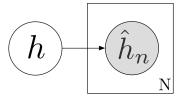

Alternatively, we can represent the $n^{\text {th }}$ measurement as

$$
\hat{h}_{n}=h+\epsilon_{n},
$$


where $\epsilon_{n}$ is the error associated with $\hat{h}_{n}$. In many contexts, it is reasonable to assume that the errors associated with $\left\{\hat{h}_{n}\right\}_{n=1}^{N}$ are wellbehaved-i.e., normally distributed, statistically unbiased, and possessing small variance $\sigma^{2}$. Under this assumption, $\epsilon_{n} \sim \mathcal{N}\left(0, \sigma^{2}\right)-$ i.e., each error is drawn from a zero-mean normal distribution with variance $\sigma^{2}$. This means that $\frac{1}{N} \sum_{n=1}^{N} \hat{h}_{n} \rightarrow h$ with probability one as $N \rightarrow \infty$-a property of a consistent estimator. Equation 1 is equivalent to the graphical model representation above provided that $\hat{h}_{n} \sim \mathcal{N}\left(h, \sigma^{2}\right)$ for $n=1, \ldots, N$. Borrowing from the economics literature, we refer to models that formalize the relationships between measurements and their errors as measurement error models.

We emphasize that even specifying a measurement error model involves making assumptions. For instance, in some contexts, the errors associated with $\left\{\hat{h}_{n}\right\}_{n=1}^{N}$ may not be not well-behaved and may even be correlated with demographic factors, such as race or gender. As an example, suppose that $\left\{\hat{h}_{n}\right\}_{n=1}^{N}$ come not from a ruler but instead from self-reports on dating websites. It might initially seem reasonable to assume that the corresponding errors are well-behaved in this context. However, Toma et al. [54] found that although men and women both over-report their height on dating websites, men are more likely to over-report and to over-report by a larger amount. Toma et al. suggest this is strategic, likely representing intentional deception. However, regardless of the cause, these errors are not well-behaved and are correlated with gender. Assuming that they are well-behaved will yield inaccurate measurements.

\subsection{Measuring Socioeconomic Status}

We now consider the process of measuring a person's socioeconomic status (SES). From a theoretical perspective, a person's SES is understood as encompassing their social and economic position in relation to others. Unlike a person's height, their SES is unobservable, so it cannot be measured directly and must instead be inferred from measurements of observable properties (and other unobservable theoretical constructs) thought to be related to it, such as income, wealth, education, and occupation. Measurements of phenomena like SES are sometimes called pragmatic measurements because they are designed to capture particular aspects of a phenomenon for particular purposes [25]. We refer to the abstraction of SES as a construct $\mathcal{S}$ and then operationalize $\mathcal{S}$ as a latent variable $s$.

The simplest way to measure a person's SES is to use an observable property-like their income-as a proxy for it. Letting the construct $\mathcal{I}$ represent the abstraction of income and operationalizing $\mathcal{I}$ as a latent variable $i$, this means specifying a both measurement model that links $s$ and $i$ and a measurement error model. For example, if we assume that $s$ and $i$ are linked via the identity function-i.e., that $s=i$-and we assume that it is possible to obtain error-free measurements of a person's income-i.e., that $\hat{i}=i-$ then $s=\hat{i}$.

Like the previous example, this example highlights that the measurement modeling process necessarily involves making assumptions. Indeed, there are many other measurement models that use income as a proxy for SES but make different assumptions about the specific relationship between them, including other deterministic models-e.g., $i=4 \times s^{2}$-and random models-e.g., $i \sim N\left(s, \sigma^{2}\right)$.

Similarly, there are many other measurement error models that make different assumptions about the errors that occur when measuring a person's income. For example, if we measure a person's monthly income by totaling the wages deposited into their account over a single one-month period, then we must use a measurement error model that accounts for the possibility that the timing of the one-month period and the timings of their wage deposits may not be aligned. Using a measurement error model that does not account for this possibility-e.g., using $\hat{i}=i$-will yield inaccurate measurements. Human Rights Watch reported exactly this scenario in the context of the Universal Credit benefits system in the U.K. [55]: The system measured a claimant's monthly income using a one-month rolling period that began immediately after they submitted their claim without accounting for the possibility described above. This meant that the system "might detect that an individual received a $£ 1000$ paycheck on March 30 and another $£ 1000$ on April 29, but not that each $£ 1000$ salary is a monthly wage [leading it] to compute the individual's benefit in May based on the incorrect assumption that their combined earnings for March and April (i.e., £2000) are their monthly wage," denying them much-needed resources.

Moving beyond income as a proxy for SES, there are arbitrarily many ways to operationalize SES via a measurement model, incorporating both measurements of observable properties, such as wealth, education, and occupation, as well as measurements of other unobservable theoretical constructs, such as cultural capital.

\subsection{Measuring Teacher Effectiveness}

We turn next to three well-known examples from the literature on fairness in computational systems, starting with the value-added models that are used by many school districts to measure the "value added" to students' academic progress by a teacher-i.e., teacher effectiveness. At the risk of stating the obvious, teacher effectiveness is an unobservable theoretical construct that cannot be measured directly and must instead be inferred from measurements of observable properties (and other unobservable theoretical constructs). Many organizations have developed models that purport to measure teacher effectiveness. For instance, SAS's Education Value-Added Assessment System (EVAAS), which is widely used across the U.S., implements two models-a multivariate response model (MRM) intended to be used when standardized tests are given to students in consecutive grades and a univariate response model intended to be used in other testing contexts. Although the models differ in terms of their mathematical details, both use changes in students' test scores (an observable property) as a proxy for teacher effectiveness.

We focus on the EVAAS MRM in this example, though we emphasize that many of the assumptions that it makes-most notably that students' test scores are a reasonable proxy for teacher effectiveness-are common to other value-added models. When describing the MRM, the EVAAS documentation states that "each teacher is assumed to be the state or district average in a specific year, subject, and grade until the weight of evidence pulls him or her above or below that average" [51]. The MRM operationalizes the effectiveness of teacher $t$ for subject $j$, grade $k$, and year ${ }^{3} l$

\footnotetext{
${ }^{3}$ Year $l$ is only present for accounting purposes; it is effectively determined by $(i, j, k)$.
} 
as a latent variable $q_{t j k l}$ via the following measurement model:

$$
\begin{aligned}
q_{t j k l} & =\mu_{j k l}+\sum_{i} \tau_{i j k l t} \\
y_{i j k l} & =\mu_{j k l}+\left(\sum_{k^{*}<=k} \sum_{t=1}^{T_{i j k^{*} l^{*}}} w_{i j k^{*} l^{*} t} \tau_{i j k^{*} l^{*} t}\right),
\end{aligned}
$$

where $\mu_{j k l}$ is the state or district's estimated mean score for subject $j$ in grade $k$ in year $l, \tau_{i j k l t} \sim \mathcal{N}\left(0, \sigma_{j k l}^{2}\right)$ is the effect of teacher $t$ on student $i$ for subject $j$ in grade $k$ in year $l, y_{i j k l}$ is student $i$ 's test score for subject $j$ in grade $k$ in year $l, w_{i j k^{*} l^{*} t}$ is the fraction of student $i$ 's instructional time attributed to teacher $t$ for subject $j$ in grade $k$ in year $l$, and $\tau_{i j k^{*} l^{*} t}$ is the effect of teacher $t$ on student $i$ for subject $j$ in grade $k^{*}$ in year $l^{*}$. The teacher effects are assumed to be normally distributed around zero, with a block diagonal covariance matrix that contains a block for each subject $j$, grade $k$, and year $l$. The MRM's measurement error model is $\hat{y}_{i j k l}=y_{i j k l}+\epsilon_{i j k l}$, where $\epsilon_{i j k l}$ is assumed to be normally distributed around zero.

As well as assuming that teacher effectiveness is fully captured by students' test scores, this model makes several other assumptions, which we make explicit here for expository purposes: 1 ) that student $i$ 's test score for subject $j$ in grade $k$ in year $l$ is a function of only their current and previous teachers' effects; 2 ) that the effectiveness of teacher $t$ for subject $j$, grade $k$, and year $l$ depends on their effects on all of their students; 3 ) that student $i$ 's instructional time for subject $j$ in grade $k$ in year $l$ may be shared between teachers; and 4 ) that a teacher may be effective in one subject but ineffective in another.

\subsection{Measuring Risk of Recidivism}

We now consider another well-known example from the literature on fairness in computational systems: the risk assessment models used in the U.S. justice system to measure a defendant's risk of recidivism. There are many such models, but we focus here on Northpointe's Correctional Offender Management Profiling for Alternative Sanctions (COMPAS), which was the subject of an investigation by Angwin et al. [4] and many academic papers [e.g., 9, 14, 34].

COMPAS draws on several criminological theories to operationalize a defendant's risk of recidivism using measurements of a variety of observable properties (and other unobservable theoretical constructs) derived from official records and interviews. These properties and measurements span four different dimensions: prior criminal history, criminal associates, drug involvement, and early indicators of juvenile delinquency problems [19]. The measurements are combined in a regression model, which outputs a score that is converted to a number between one and ten with ten being the highest risk. Although the full mathematical details of COMPAS are not readily available, the COMPAS documentation mentions numerous assumptions, the most important of which is that recidivism is defined as "a new misdemeanor or felony arrest within two years." We discuss the implications of this assumption in sections 3 and 4 .

\subsection{Measuring Patient Benefit}

Finally, we turn to a different type of risk assessment model, used in the U.S. healthcare system to identify the patients that will benefit the most from enrollment in high-risk care management programsi.e., programs that provide access to additional resources for patients with complex health issues. As explained by Obermeyer et al., these models assume that "those with the greatest care needs will benefit the most from the programs" [43]. Furthermore, many of them operationalize greatest care needs as greatest care costs.

This assumption-i.e., that care costs are a reasonable proxy for care needs-transforms the difficult task of measuring the extent to which a patient will benefit from a program (an unobservable theoretical construct) into the simpler task of predicting their future care costs based on their past care costs (an observable property). However, this assumption masks an important confounding factor: patients with comparable past care needs but different access to care will likely have different past care costs. As we explain in the next section, even without considering any other details of these models, this assumption can lead to fairness-related harms [43].

\section{TESTING ASSUMPTIONS}

As we explained in the previous section, the measurement modeling process necessarily involves making assumptions. However, these assumptions must be made explicit and tested before the resulting measurements are used. Leaving them implicit or untested obscures any possible mismatches between the theoretical understanding of the construct purported to be measured and its operationalization, in turn obscuring any resulting fairness-related harms. In this section, we show how measurement modeling provides a set of tools for surfacing such mismatches. These tools differentiate measurement modeling from statistical modeling and machine learning as they are typically used in computer science. We therefore argue that it is these tools that give measurement modeling its power as a framework for understanding fairness in computational systems.

In contrast to computer scientists, who focus primarily on out-ofsample prediction, quantitative social scientists typically test their assumptions by assessing construct reliability and construct validity. Quinn et al. describe these concepts as follows: "The evaluation of any measurement is generally based on its reliability (can it be repeated?) and validity (is it right?). Embedded within the complex notion of validity are interpretation (what does it mean?) and application (does it 'work?')" [49]. We contribute fairness-oriented conceptualizations of construct reliability and construct validity that unite traditions from political science, education, and psychology by drawing on the work of Quinn et al. [49], Jackman [30], Messick [40], and Loevinger [36], among others. We illustrate these conceptualizations using the five examples introduced in the previous section, arguing that they constitute a set of tools that will enable researchers and practitioners to 1) better anticipate fairness-related harms that can be obscured by focusing primarily on out-of-sample prediction, and 2) identify potential causes of fairness-related harms in ways that reveal concrete, actionable avenues for mitigating them.

\subsection{Construct Reliability}

We start by describing construct reliability-a concept that is roughly analogous to the concept of precision (i.e., the inverse of variance) in statistics [30]. Assessing construct reliability means answering the following question: do similar inputs to a measurement model, possibly presented at different points in time, yield similar outputs? 
If the answer to this question is no, then the model lacks reliability, meaning that we may not want to use its measurements. We note that a lack of reliability can also make it challenging to assess construct validity. Although different disciplines emphasize different aspects of construct reliability, we argue that there is one aspectnamely test-retest reliability, which we describe below-that is especially relevant in the context of fairness in computational systems. ${ }^{4}$

3.1.1 Test-retest reliability. Test-retest reliability refers to the extent to which measurements of an unobservable theoretical construct, obtained from a measurement model at different points in time, remain the same, assuming that the construct has not changed.

For example, when measuring a person's height, operationalized as the length from the bottom of their feet to the top of their head when standing erect, measurements that vary by several inches from one day to the next would suggest a lack of test-retest reliability. Investigating this variability might reveal its cause to be the assumption that a person's shoes should contribute to their height.

As another example, many value-added models, including the EVAAS MRM, have been criticized for their lack of test-retest reliability. For instance, in Weapons of Math Destruction [46], O'Neil described how value-added models often produce measurements of teacher effectiveness that vary dramatically between years. In one case, she described Tim Clifford, an accomplished and respected New York City middle school teacher with over 26 years of teaching experience. For two years in a row, Clifford was evaluated using a value-added model, receiving a score of 6 out of 100 in the first year, followed by a score of 96 in the second. It is extremely unlikely that teacher effectiveness would vary so dramatically from one year to the next. Instead, this variability, which suggests a lack of test-retest reliability, points to a possible mismatch between the construct purported to be measured and its operationalization.

As a third example, had the developers of the Universal Credit benefits system described in section 2.2 assessed the test-retest reliability of their system by checking that the system's measurements of a claimant's income were the same no matter when their one-month rolling period began, they might have anticipated (and even mitigated) the harms revealed by Human Rights Watch [55].

Finally, we note that an apparent lack of test-retest reliability does not always point to a mismatch between the theoretical understanding of the construct purported to be measured and its operationalization. In some cases, an apparent lack of test-retest reliability can instead be the result of unexpected changes to the construct itself. For example, although we typically think of a person's height as being something that remains relatively static over the course of their adult life, most people actually get shorter as they get older.

\footnotetext{
${ }^{4}$ Inter-rater reliability refers to the extent to which multiple raters, experts, judges, or annotators agree in their assessments (i.e., outputs) when given the same task (i.e. inputs). Although inter-rater reliability plays a key role when measuring unobservable theoretical constructs via qualitative methods, it is less immediately relevant when using quantitative methods. For this reason, we omit inter-rater reliability from our conceptualization of construct reliability. We also omit inter-item reliability, which refers to the extent to which the inputs to a measurement model are correlated with one another. Although we believe that inter-item reliability may be relevant in the context of fairness in computational systems, we could not find any examples of fairnessrelated harms that would likely have been surfaced by assessing inter-item reliability.
}

\subsection{Construct Validity}

Whereas construct reliability is roughly analogous to the concept of precision in statistics, construct validity is roughly analogous to the concept of statistical unbiasedness [30]. Establishing construct validity means demonstrating, in a variety of ways, that the measurements obtained from measurement model are both meaningful and useful: Does the operationalization capture all relevant aspects of the construct purported to be measured? Do the measurements look plausible? Do they correlate with other measurements of the same construct? Or do they vary in ways that suggest that the operationalization may be inadvertently capturing aspects of other constructs? Are the measurements predictive of measurements of any relevant observable properties (and other unobservable theoretical constructs) thought to be related to the construct, but not incorporated into the operationalization? Do the measurements support known hypotheses about the construct? What are the consequences of using the measurements-including any societal impacts [40, 52]. We emphasize that a key feature, not a bug, of construct validity is that it is not a yes/no box to be checked: construct validity is always a matter of degree, to be supported by critical reasoning [36].

Different disciplines have different conceptualizations of construct validity, each with its own rich history. For example, in some disciplines, construct validity is considered distinct from content validity and criterion validity, while in other disciplines, content validity and criterion validity are grouped under the umbrella of construct validity. Our conceptualization unites traditions from political science, education, and psychology by bringing together the seven different aspects of construct validity that we describe below. We argue that each of these aspects plays a unique and important role in understanding fairness in computational systems.

3.2.1 Face validity. Face validity refers to the extent to which the measurements obtained from a measurement model look plausiblea "sniff test" of sorts. This aspect of construct validity is inherently subjective, so it is often viewed with skepticism if it is not supplemented with other, less subjective evidence. However, face validity is a prerequisite for establishing construct validity: if the measurements obtained from a measurement model aren't facially valid, then they are unlikely to possess other aspects of construct validity.

It is likely that the models described in section 2 would yield measurements that are, for the most part, facially valid. For example, measurements obtained by using income as a proxy for SES would most likely possess face validity. SES and income are certainly related and, in general, a person at the high end of the income distribution (e.g., a CEO) will have a different SES than a person at the low end (e.g., a barista). Similarly, given that COMPAS draws on several criminological theories to operationalize a defendant's risk of recidivism, it is likely that the resulting scores would look plausible.

One exception to this pattern is the EVAAS MRM. Some scores may look plausible-after all, students' test scores are not unrelated to teacher effectiveness-but the dramatic variability that we described above in the context of test-retest reliability is implausible.

3.2.2 Content validity. Content validity refers to the extent to which an operationalization wholly and fully captures the substantive nature of the construct purported to be measured. This aspect of construct validity has three sub-aspects, which we describe below. 
The first sub-aspect relates to the construct's contestedness. If a construct is essentially contested then it has multiple contextdependent, and sometimes even conflicting, theoretical understandings. Contestedness makes it inherently hard to assess content validity: if a construct has multiple theoretical understandings, then it is unlikely that a single operationalization can wholly and fully capture its substantive nature in a meaningful fashion. For this reason, some traditions make a single theoretical understanding of the construct purported to be measured a prerequisite for establishing content validity $[25,30]$. However, other traditions simply require an articulation of which understanding is being operationalized [53]. We take the perspective that the latter approach is more practical because it is often the case that unobservable theoretical constructs are essentially contested, yet we still wish to measure them. Indeed, fairness itself is one such construct, as we explain in section 4 .

Of the models described in section 2, most are intended to measure unobservable theoretical constructs that are (relatively) uncontested. One possible exception is patient benefit, which can be understood in a variety of different ways. However, the understanding that is operationalized in most high-risk care management enrollment models is clearly articulated. As Obermeyer et al. explain, "[the patients] with the greatest care needs will benefit the most" from enrollment in high-risk care management programs [43].

The second sub-aspect of content validity is sometimes known as substantive validity. This sub-aspect moves beyond the theoretical understanding of the construct purported to be measured and focuses on the measurement modeling process-i.e., the assumptions made when moving from abstractions to mathematics. Establishing substantive validity means demonstrating that the operationalization incorporates measurements of those-and only those-observable properties (and other unobservable theoretical constructs, if appropriate) thought to be related to the construct.

For example, although a person's income contributes to their SES, their income is by no means the only contributing factor. Wealth, education, and occupation all affect a person's SES, as do other unobservable theoretical constructs, such as cultural capital. For instance, an artist with significant wealth but a low income should have a higher SES than would be suggested by their income alone.

As another example, COMPAS defines recidivism as "a new misdemeanor or felony arrest within two years.” By assuming that arrests are a reasonable proxy for crimes committed, COMPAS fails to account for false arrests or crimes that do not result in arrests [50]. Indeed, no computational system can ever wholly and fully capture the substantive nature of crime by using arrest data as a proxy.

Similarly, high-risk care management enrollment models assume that care costs are a reasonable proxy for care needs. However, a patient's care needs reflect their underlying health status, while their care costs reflect both their access to care and their health status.

Finally, establishing structural validity, the third sub-aspect of content validity, means demonstrating that the operationalization captures the structure of the relationships between the incorporated observable properties (and other unobservable theoretical constructs, if appropriate) and the construct purported to be measured, as well as the interrelationships between them [36, 40].

In addition to assuming that teacher effectiveness is wholly and fully captured by students' test scores-a clear threat to substantive validity [2]-the EVAAS MRM assumes that a student's test score for subject $j$ in grade $k$ in year $l$ is approximately equal to the sum of the state or district's estimated mean score for subject $j$ in grade $k$ in year $l$ and the student's current and previous teachers' effects (weighted by the fraction of the student's instructional time attributed to each teacher). However, this assumption ignores the fact that, for many students, the relationship may be more complex.

3.2.3 Convergent validity. Convergent validity refers to the extent to which the measurements obtained from a measurement model correlate with other measurements of the same construct, obtained from measurement models for which construct validity has already been established. This aspect of construct validity is typically assessed using quantitative methods, though doing so can reveal qualitative differences between different operationalizations.

We note that assessing convergent validity raises an inherent challenge: "If a new measure of some construct differs from an established measure, it is generally viewed with skepticism. If a new measure captures exactly what the previous one did, then it is probably unnecessary" [49]. The measurements obtained from a new measurement model should therefore deviate only slightly from existing measurements of the same construct. Moreover, for the model to be viewed as possessing convergent validity, these deviations must be well justified and supported by critical reasoning.

Many value-added models, including the EVAAS MRM, lack convergent validity [2]. For example, in Weapons of Math Destruction [46], O’Neil described Sarah Wysocki, a fifth-grade teacher who received a low score from a value-added model despite excellent reviews from her principal, her colleagues, and her students' parents.

As another example, measurements of SES obtained from the model described in section 2.2 and measurements of SES obtained from the National Committee on Vital and Health Statistics would likely correlate somewhat because both operationalizations incorporate income. However, the latter operationalization also incorporates measurements of other observable properties, including wealth, education, occupation, economic pressure, geographic location, and family size [45]. As a result, it is also likely that there would also be significant differences between the two sets of measurements. Investigating these differences might reveal aspects of the substantive nature of SES, such as wealth or education, that are missing from the model described in section 2.2. In other words, and as we described above, assessing convergent validity can reveal qualitative differences between different operationalizations of a construct.

We emphasize that assessing the convergent validity of a measurement model using measurements obtained from measurement models that have not been sufficiently well validated can yield a false sense of security. For example, scores obtained from COMPAS would likely correlate with scores obtained from other models that similarly use arrests as a proxy for crimes committed, thereby obscuring the threat to content validity that we described above.

3.2.4 Discriminant validity. Discriminant validity refers to the extent to which the measurements obtained from a measurement model vary in ways that suggest that the operationalization may be inadvertently capturing aspects of other constructs. Measurements of one construct should only correlate with measurements of another to the extent that those constructs are themselves related. As a special case, if two constructs are totally unrelated, then there should be no correlation between their measurements [25]. 
Establishing discriminant validity can be especially challenging when a construct has relationships with many other constructs. SES, for example, is related to almost all social and economic constructs, albeit to varying extents. For instance, SES and gender are somewhat related due to labor segregation and the persistent gender wage gap, while SES and race are much more closely related due to historical racial inequalities resulting from structural racism. When assessing the discriminant validity of the model described in section 2.2, we would therefore hope to find correlations that reflect these relationships. If, however, we instead found that the resulting measurements were perfectly correlated with gender or uncorrelated with race, this would suggest a lack of discriminant validity.

As another example, Obermeyer et al. found a strong correlation between measurements of patients' future care needs, operationalized as future care costs, and race [43]. According to their analysis of one model, only $18 \%$ of the patients identified for enrollment in highrisk care management programs were Black. This correlation contradicts expectations. Indeed, given the enormous racial health disparities in the U.S., we might even expect to see the opposite pattern. Further investigation by Obermeyer et al. revealed that this threat to discriminant validity was caused by the confounding factor that we described in section 2.5: Black and white patients with comparable past care needs had radically different past care costs-a consequence of structural racism that was then exacerbated by the model.

3.2.5 Predictive validity. Predictive validity refers to the extent to which the measurements obtained from a measurement model are predictive of measurements of any relevant observable properties (and other unobservable theoretical constructs) thought to be related to the construct purported to be measured, but not incorporated into the operationalization. Assessing predictive validity is therefore distinct from out-of-sample prediction [24, 41]. Predictive validity can be assessed using either qualitative or quantitative methods. We note that in contrast to the aspects of construct validity that we discussed above, predictive validity is primarily concerned with the utility of the measurements, not their meaning.

As a simple illustration of predictive validity, taller people generally weigh more than shorter people. Measurements of a person's height should therefore be somewhat predictive of their weight.

Similarly, a person's SES is related to many observable propertiesranging from purchasing behavior to media appearances-that are not always incorporated into models for measuring SES. Measurements obtained by using income as a proxy for SES would most likely be somewhat predictive of many of these properties, at least for people at the high and low ends of the income distribution.

We note that the relevant observable properties (and other unobservable theoretical constructs) need not be "downstream" of (i.e., thought to be influenced by) the construct. Predictive validity can also be assessed using "upstream" properties and constructs, provided that they are not incorporated into the operationalization. For example, Obermeyer et al. investigated the extent to which measurements of patients' future care needs, operationalized as future care costs, were predictive of patients' health statuses (which were not part of the model that they analyzed) [43]. They found that Black and white patients with comparable future care costs did not have comparable health statuses-a threat to predictive validity caused (again) by the confounding factor described in section 2.5.
3.2.6 Hypothesis validity. Hypothesis validity refers to the extent to which the measurements obtained from a measurement model support substantively interesting hypotheses about the construct purported to be measured. Much like predictive validity, hypothesis validity is primarily concerned with the utility of the measurements. We note that the main distinction between predictive validity and hypothesis validity hinges on the definition of "substantively interesting hypotheses." As a result, the distinction is not always clear cut. For example, is the hypothesis "People with higher SES are more likely to be mentioned in the New York Times" sufficiently substantively interesting? Or would it be more appropriate to use the hypothesized relationship to assess predictive validity? For this reason, some traditions merge predictive and hypothesis validity [e.g., 30].

Turning again to the value-added models discussed in section 2.3, it is extremely unlikely that the dramatically variable scores obtained from such models would support most substantively interesting hypotheses involving teacher effectiveness, again suggesting a possible mismatch between the theoretical understanding of the construct purported to be measured and its operationalization.

Using income as a proxy for SES would likely support somethough not all-substantively interesting hypotheses involving SES. For example, many social scientists have studied the relationship between SES and health outcomes, demonstrating that people with lower SES tend to have worse health outcomes. Measurements of SES obtained from the model described in section 2.2 would likely support this hypothesis, albeit with some notable exceptions. For instance, wealthy college students often have low incomes but good access to healthcare. Combined with their young age, this means that they typically have better health outcomes than other people with comparable incomes. Examining these exceptions might reveal aspects of the substantive nature of SES, such as wealth and education, that are missing from the model described in section 2.2.

3.2.7 Consequential validity. Consequential validity, the final aspect in our fairness-oriented conceptualization of construct validity, is concerned with identifying and evaluating the consequences of using the measurements obtained from a measurement model, including any societal impacts. Assessing consequential validity often reveals fairness-related harms. Consequential validity was first introduced by Messick, who argued that the consequences of using the measurements obtained from a measurement model are fundamental to establishing construct validity [40]. This is because the values that are reflected in those consequences both derive from and contribute back the theoretical understanding of the construct purported to be measured. In other words, the "measurements both reflect structure in the natural world, and impose structure upon it," [26]-i.e., the measurements shape the ways that we understand the construct itself. Assessing consequential validity therefore means answering the following questions: How is the world shaped by using the measurements? What world do we wish to live in? If there are contexts in which the consequences of using the measurements would cause us to compromise values that we wish to uphold, then the measurements should not be used in those contexts.

For example, when designing a kitchen, we might use measurements of a person's standing height to determine the height at which to place their kitchen countertop. However, this may render the countertop inaccessible to them if they use a wheelchair. 
As another example, because the Universal Credit benefits system described in section 2.2 assumed that measuring a person's monthly income by totaling the wages deposited into their account over a single one-month period would yield error-free measurements, many people-especially those with irregular pay schedulesreceived substantially lower benefits than they were entitled to.

The consequences of using scores obtained from value-added models are well described in the literature on fairness in computational systems. Many school districts have used such scores to make decisions about resource distribution and even teachers' continued employment, often without any way to contest these decisions [2, 3]. In turn, this has caused schools to manipulate their scores and encouraged teachers to "teach to the test," instead of designing more diverse and substantive curricula [46]. As well as the cases described above in sections 3.1.1 and 3.2.3, in which teachers were fired on the basis of low scores despite evidence suggesting that their scores might be inaccurate, Amrein-Beardsley and Geiger [3] found that EVAAS consistently gave lower scores to teachers at schools with higher proportions of non-white students, students receiving special education services, lower-SES students, and English language learners. Although it is possible that more effective teachers simply chose not to teach at those schools, it is far more likely that these lower scores reflect societal biases and structural inequalities. When scores obtained from value-added models are used to make decisions about resource distribution and teachers' continued employment, these biases and inequalities are then exacerbated.

The consequences of using scores obtained from COMPAS are also well described in the literature on fairness in computational systems, most notably by Angwin et al. [4], who showed that COMPAS incorrectly scored Black defendants as high risk more often than white defendants, while incorrectly scoring white defendants as low risk more often than Black defendants. By defining recidivism as "a new misdemeanor or felony arrest within two years," COMPAS fails to account for false arrests or crimes that do not result in arrests. This assumption therefore encodes and exacerbates racist policing practices, leading to the racial disparities uncovered by Angwin et al. Indeed, by using arrests as a proxy for crimes committed, COMPAS can only exacerbate racist policing practices, rather than transcending them $[7,13,23,37,39]$. Furthermore, the COMPAS documentation asserts that "the COMPAS risk scales are actuarial risk assessment instruments. Actuarial risk assessment is an objective method of estimating the likelihood of reoffending. An individual's level of risk is estimated based on known recidivism rates of offenders with similar characteristics" [19]. By describing COMPAS as an "objective method," Northpointe misrepresents the measurement modeling process, which necessarily involves making assumptions and is thus never objective. Worse yet, the label of objectiveness obscures the organizational, political, societal, and cultural values that are embedded in COMPAS and reflected in its consequences.

Finally, we return to the high-risk care management models described in section 2.5. By operationalizing greatest care needs as greatest care costs, these models fail to account for the fact that patients with comparable past care needs but different access to care will likely have different past care costs. This omission has the greatest impact on Black patients. Indeed, when analyzing one such model, Obermeyer et al. found that only $18 \%$ of the patients identified for enrollment were Black [43]. In addition, Obermeyer et al. found that Black and white patients with comparable future care costs did not have comparable health statuses. In other words, these models exacerbate the enormous racial health disparities in the U.S. as a consequence of a seemingly innocuous assumption.

\section{FAIRNESS AS A CONSTRUCT}

We now explain how measurement modeling can contribute to recent debates about fairness definitions. Although fairness feels instinctively different to the constructs discussed so far, it is an unobservable theoretical construct, albeit one about which there have been millennia of disagreements. These disagreements reflect the fact that fairness is an essentially contested construct [21, 42]i.e., fairness has multiple context-dependent, and sometimes even conflicting, theoretical understandings. The contested nature of fairness makes it inherently hard to measure: As we described in section 3.2.2, some traditions make a single theoretical understanding a prerequisite for establishing content-and hence construct-validity. Other traditions (which we draw on in our fairness-oriented conceptualization of construct validity) abandon this prerequisite and instead simply require an articulation of which understanding is being operationalized, arguing that a failure to do this makes it difficult to meaningfully compare different operationalizations.

We start by discussing aspects of the substantive nature of fairness that are necessarily missing from the quantitative, parity-based operationalizations commonly found in the literature on fairness in computational systems, focusing on the resulting threats to both content and convergent validity. We then draw on well-known examples from this literature to argue that although recent debates about fairness definitions have been framed in terms of operationalizations, they are, in fact, debates about different theoretical understandings of fairness-i.e., debates about values. We argue that by framing these debates in terms of operationalizations, they are rendered less accessible to the stakeholders that are most likely to be affected by the computational systems in question. Finally, we touch briefly on the role of demographic factors in measuring fairness.

\subsection{The Substantive Nature of Fairness}

To date, much of the literature on fairness in computational systems has focused primarily on quantitative, parity-based operationalizations of fairness [e.g., 17, 20, 28]. These operationalizations are appealing to computer scientists because of their quantitative nature and because they operate within the boundaries of a single computational system, without reference to the broader societal context in which the system is situated [38]. Yet these same properties mean that these operationalizations necessarily lack aspects of the substantive nature of fairness found in many of the theoretical understandings long discussed by philosophers, lawyers, and social scientists. For example, Arneson [5] presents four different, and sometimes conflicting, theoretical understandings of equal opportunity-itself an understanding of fairness. Of these understandings, it might be possible to operationalize at most one or two using quantitative, parity-based methods. Operationalizing the others using such methods would lead to a lack of content validity.

We note that many theoretical understandings of fairness, including some of the understandings discussed by Arneson [5], depend 
on some notion of justice, such as procedural justice, distributive justice, or representational justice. Any operationalization of fairness that omits justice entirely cannot therefore be thought of as wholly and fully capturing the substantive nature of these understandings. Moreover, any such operationalization would likely be ineffective at remedying historical injustices-a threat to consequential validity.

\subsection{Individual Fairness vs. Group Fairness}

Putting aside the threats posed to content and consequential validity by quantitative, parity-based operationalizations of fairness, we turn next to the recent debates about fairness definitions found in the literature on fairness in computational systems. In what was arguably the first such debate, Dwork et al. [17] contrasted individual fairness, which requires that similar people be treated similarly, and group fairness, which requires that different groups of people, such as groups defined in terms of different demographic factors, be treated similarly. Dwork et al. argued that computational systems that satisfy some definition of group fairness can still yield fairnessrelated harms for people belonging to those groups. This argument has been discussed in many papers since then [e.g., 12, 13, 32], forming an ongoing debate about individual fairness and group fairness.

Although this debate is usually framed in terms of mathematicsi.e., in terms of operationalizations-it is, at its core, a debate about different theoretical understandings of fairness. Viewing the debate through the lens of measurement modeling offers a new way to engage with it. Individual fairness and group fairness are conflicting theoretical understandings of fairness. In contrast to the former understanding, the latter understanding fails to account for fairnessrelated harms experienced by individual people. Any operationalization of the latter understanding will necessarily lack content validity if it is viewed as an operationalization of the former. The debate is therefore about values-i.e., to what extent should individual experiences matter?-not about the measurement modeling process.

\subsection{Individual Fairness}

Even debates about different definitions of individual fairness are often debates about different theoretical understandings. Individual fairness requires that similar people be treated similarly. However, the similarity of two people is itself an essentially contested construct, with multiple context-dependent theoretical understandings. Moreover, even when focusing on a single understanding, measuring the similarity of two people is a nontrivial undertaking that involves many different aspects of the human experience, including aspects that are inherently subjective or personal. Any practical operationalization of similarity will therefore likely lack contentand possibly even consequential-validity [29]. Yet academic papers about individual fairness often obscure or downplay this reality.

For example, although Dwork et al. acknowledge that similarity may not be easy to measure, they argue that "the [similarity] metric may reflect the 'best' available approximation as agreed upon by society" [17]. Despite giving an implicit nod to the assumptions involved in the measurement modeling process, including the assumption of which theoretical understanding to operationalize, this definition obscures the difficulty of making explicit and testing those assumptions by using the phrase "as agreed upon by society."

As another example, Joseph et al. present a framework for "ensuring" individual fairness when using a computational system to decide who should receive some resource or opportunity [31, 32]. Crucially, their framework assumes that recipients should be selected the basis of their quality, which is further assumed to be observable and easy to measure directly, yielding error-free measurements: "[O]ur definition of fairness... assumes the existence of an accurate mapping from features to true quality for the task at hand" [31]. This assumption downplays the fact that quality is an essentially contested construct, in turn downplaying the difficulty of measuring the similarity of two people in terms of their quality.

\subsection{Group Fairness}

Like debates about individual fairness, debates about different definitions of group fairness are often debates about different theoretical understandings. The most well-known debate about group fairness involves COMPAS, which operationalizes a defendant's risk of recidivism using measurements of a variety of observable properties (and other unobservable theoretical constructs), as we described in section 2.4. The debate began when Angwin et al. [4] showed that COMPAS incorrectly scored Black defendants as high risk more often than white defendants, while incorrectly scoring white defendants as low risk more often than Black defendants. In other words, Angwin et al. showed that COMPAS was unfair because it lacked error-rate balance. ${ }^{5}$ Northpointe responded by arguing that COMPAS was fair because the probability of recidivism among Black defendants scored as high risk was the same as the probability of recidivism among white defendants scored as high risk-i.e., COMPAS possessed predictive parity. Prompted by this, many academic papers began to discuss the properties and shortcomings of different definitions of group fairness, with some papers demonstrating that it is impossible for a system like COMPAS to possess both error-rate balance and predictive parity when the probability of recidivism for Black defendants is not the same as the probability of recidivism for white defendants [9, 11, 13, 15, 34, 48].

Despite being framed in terms of operationalizations, this debate is actually about different theoretical understandings of fairness. Indeed, in their discussion of the debate, Corbett et al. wrote, "At the heart of [the] disagreement is a subtle ethical question: What does it mean for an algorithm to be fair?" [14]. In other words, error-rate balance and predictive parity are operationalizations of different theoretical understandings of group fairness. These understandingsand hence their operationalizations-have very different consequences. Predictive parity means that a score has the same meaning when it is given to a Black defendant as it does when it is given to a white defendant. In contrast, error-rate balance means that the errors experienced by Black defendants and white defendants are comparable. Choosing one of these theoretical understandings over the other therefore means choosing which values we wish to uphold. But, by couching the debate in mathematics, this choice is concealed from the stakeholders that are most likely to be affected by it.

\subsection{Demographic Factors}

Group fairness, as we explained in section 4.2, requires that different groups of people, such as groups defined in terms of different demographic factors, be treated similarly. But many demographic factors, such as race or gender, are themselves essentially contested constructs, with theoretical understandings that vary across cultures

\footnotetext{
${ }^{5}$ Error-rate balance is also known as equalized odds [28].
} 
and over time. As a result, measuring group fairness often requires that we first undertake the non-trivial task of measuring these constructs. Ideally, measurements of these constructs should be self reported, thereby limiting the potential for harmful errors. However, there are many contexts in which self-reports at the level of individual people are not available or readily accessible. In these contexts, it may be tempting to infer the relevant constructs from measurements of observable properties (and other unobservable theoretical constructs) thought to be related to them-i.e., operationalize them via a measurement model. We emphasize that even with careful consideration of different theoretical understandings and careful assessment of both construct reliability and construct validity, this approach is fraught and likely to cause a variety of fairness-related and other harms [e.g., 8, 22, 33, 35, 44], despite good intentions. In other words, the consequences of using the resulting measurements will likely cause us to compromise values that we wish to uphold.

\section{DISCUSSION}

Many computational systems involve unobservable theoretical constructs-abstractions that describe phenomena of theoretical interest, such as socioeconomic status, teacher effectiveness, and risk of recidivism. Because these constructs cannot be measured directly, they must instead be inferred from measurements of observable properties (and other unobservable theoretical constructs) thought to be related to them. However, this process-the measurement modeling process-necessarily involves making assumptions, thereby introducing the potential for mismatches between the theoretical understanding of the construct purported to be measured and its operationalization. As we argued in section 3, many of the harms studied in the literature on fairness in computational systems are direct results of such mismatches. This is because the assumptions made when moving from abstractions to mathematics often encode and exacerbate societal biases and structural inequalities.

Although measurement modeling is fundamental to the quantitative social sciences, it has not traditionally played a role in computer science. As a result, researchers and practitioners are often inclined to collapse the distinctions between constructs and their operationalizations, either colloquially or epistemically. But collapsing these distinctions removes opportunities to anticipate and mitigate fairness-related harms by eliding the space in which they are most often introduced. Further compounding this issue is the fact that measurements of unobservable theoretical constructs are often treated as if they were obtained directly and without errors-i.e., a source of ground truth. Measurements end up standing in for the constructs purported to be measured, normalizing the assumptions made during the measurement modeling process and embedding them throughout society. In other words, "measures are more than a creation of society, they create society" [1]-a view also expressed by Bowker and Star in their exploration of the consequences of classification in computing systems [10]. ${ }^{6}$ Collapsing the distinctions

\footnotetext{
${ }^{6}$ Bowker and Star express the view that measurements create society via their discussion of classification in computational systems [10]. To see how classification in computational systems subtly but fundamentally creates categories and stratifications in the world, consider a user creating an account on a website. The website might require the user to select either "Male" or "Female" as their gender, refusing to create the user's account if one of these options is not selected. Bowker and Star argue that these kinds of design choices are fundamentally political: "Seemingly purely technical issues like how to name things and how to store data in fact constitute much of human interaction
}

between constructs and their operationalizations is therefore not just theoretically or pedantically concerning-it is practically concerning with very real, fairness-related consequences. Moreover, because most computational systems are developed by computer scientists, the practice of collapsing these distinctions is widespread [47].

We argue that measurement modeling provides a both a language for articulating the distinctions between constructs and their operationalizations and set of tools-namely construct reliability and construct validity-for surfacing possible mismatches. In section 3, we therefore proposed fairness-oriented conceptualizations of construct reliability and construct validity, uniting traditions from political science, education, and psychology. We showed how these conceptualizations can be used to 1) anticipate fairness-related harms that can be obscured by focusing primarily on out-of-sample prediction, and 2) identify potential causes of fairness-related harms in ways that reveal concrete, actionable avenues for mitigating them. We acknowledge that assessing construct reliability and construct validity can be time-consuming. However, ignoring them means that we run the risk of creating a world that we do not wish to live in.

Finally, we turned to fairness itself, highlighting aspects of the substantive nature of fairness that are missing from the quantitative, parity-based operationalizations commonly found in the literature on fairness in computational systems. We argue that although recent debates about fairness definitions have been framed in terms of operationalizations, they are, in fact, debates about different theoretical understandings of fairness-i.e., debates about values. Moreover, many definitions of fairness involve other essentially contested constructs, such as similarity, quality, and even demographic factors like race or gender. These relationships are therefore reminiscent of the "layers of bias" described by Eckhouse et al. [18] in the context of risk assessment models: "[E] ach layer depends on the layers below it: Without assurances about the foundational layers, the fairness of the top layers is irrelevant." Worse yet, by using such fairness definitions to label computational systems as "fair," we risk the adoption of these systems without any critical assessment because their fairness is assumed to be guaranteed.

To conclude, measurement modeling promotes greater transparency and accountability by providing researchers and practitioners with a set of tools for making explicit and testing assumptions. By using these tools to surface mismatches between constructs and their operationalizations, researchers will be better able to anticipate and mitigate fairness-related harms arising from computational systems. Although measurement modeling is largely missing from computer science, we argue that it should be essential knowledge for everyone developing or deploying computational systems.

\section{ACKNOWLEDGMENTS}

We thank Solon Barocas, Su Lin Blodgett, Alex Chouldechova, Hal Daumé, Lise Getoor, Moritz Hardt, Josh Kroll, Alexandra Olteanu, Forough Poursabzi-Sangdeh, Brandon Stewart, Philip Thomas, Jenn Wortman Vaughan, and many others for feedback on this paper.

\section{REFERENCES}

[1] Ken Alder. 2002. The measure of all things: The seven-year odyssey and hidden error that transformed the world.

and much of what we come to know as natural." As another example, many researchers have argued that race as a category further entrenches structural racism [e.g., 7, 27]. 
[2] Audrey Amrein-Beardsley. 2008. Methodological concerns about the Education Value-Added Assessment System. Educational Researcher 37, 2 (2008), 65-75.

[3] Audrey Amrein-Beardsley and Tray Geiger. 2020. Methodological Concerns About the Education Value-Added Assessment System (EVAAS): Validity, Reliability, and Bias. SAGE Open 10, 2 (2020), 2158244020922224.

[4] Julia Angwin, Jeff Larson, Surya Mattu, and Lauren Kirchner. 2016. Machine Bias. ProPublica (May 2016)

[5] Richard Arneson. 2018. Four conceptions of equal opportunity. The Economic fournal 128, 612 (2018), F152-F173.

[6] Solon Barocas, Kate Crawford, Aaron Shapiro, and Hanna Wallach. 2017. The problem with bias: from allocative to representational harms in machine learning. Special Interest Group for Computing. Information and Society (SIGCIS) (2017).

[7] Ruha Benjamin. 2019. Race after Technology: Abolitionist Tools for the New fim Code.

[8] Cynthia Bennett and Os Keyes. 2020. What is the point of fairness? Interactions 27,3 (2020), 35-39.

[9] Richard Berk, Hoda Heidari, Shahin Jabbari, Michael Kearns, and Aaron Roth 2018. Fairness in criminal justice risk assessments: the state of the art. Soc. Meth. Res. (2018). https://doi.org/10.1177/0049124118782533

[10] Geoffrey C Bowker and Susan Leigh Star. 2000. Sorting things out: Classification and its consequences. MIT Press.

[11] Alexandra Chouldechova. 2017. Fair prediction with disparate impact: A study of bias in recidivism prediction instruments. Big Data 5, 2 (2017), 153-163.

[12] Alexandra Chouldechova and Aaron Roth. 2018. The frontiers of fairness in machine learning. arXiv preprint arXiv:1810.08810 (2018)

[13] Sam Corbett-Davies and Sharad Goel. 2018. The Measure and Mismeasure of Fairness: A Critical Review of Fair Machine Learning. Preprint, arXiv:1808.00023 (2018).

[14] Sam Corbett-Davies, Emma Pierson, Avi Feller, and Sharad Goel. 2016. A computer program used for bail and sentencing decisions was labeled biased against blacks. It's actually not that clear. Washington Post (October 2016).

[15] Sam Corbett-Davies, Emma Pierson, Avi Feller, Sharad Goel, and Aziz Huq. 2017. Algorithmic decision making and the cost of fairness.

[16] Kate Crawford. 2017. The Trouble with Bias. NIPS Keynote, https://www. youtube.com/watch?v=fMym_BKWQzk, accessed 08-22-2018.

[17] Cynthia Dwork, Moritz Hardt, Toniann Pitassi, Omer Reingold, and Richard Zemel. 2012. Fairness through awareness. ITCS (2012).

[18] Laurel Eckhouse, Kristian Lum, Cynthia Conti-Cook, and Julie Ciccolini. 2019 Layers of bias: A unified approach for understanding problems with risk assessment. Criminal Fustice and Behavior 46, 2 (2019), 185-209.

[19] equivant. 2017. Practitioner's Guide to COMPAS Core. http://www.equivant. com/assets/img/content/Practitioners_Guide_COMPASCore_121917.pdf http://www.equivant.com/assets/img/content/Practitioners_Guide_ COMPASCore 121917.pdf, accessed 08-22-2018.

[20] Sorelle A Friēler, Carlos Scheidegger, and Suresh Venkatasubramanian. 2016 On the (im)possibility of fairness. Preprint, arXiv:1609.07236 (2016).

[21] Walter Bryce Gallie. 1955. Essentially contested concepts. In Proceedings of the Aristotelian Society, Vol. 56. JSTOR, 167-198.

[22] Jake Goldenfein. 2019. The Profiling Potential of Computer Vision and the Challenge of Computational Empiricism. In Proc. 2019 ACM FAT* Conference.

[23] Ben Green. 2020. The false promise of risk assessments: epistemic reform and the limits of fairness. In Proceedings of the 2020 Conference on Fairness, Accountability, and Transparency. 594-606.

[24] Justin Grimmer. 2012. Comment: Evaluating model performance in fictitious prediction problems. Technical Report.

[25] David J Hand. 2004. Measurement theory and practice. Arnold, Hodder Headline.

[26] David J Hand. 2016. Measurement: A Very Short Introduction. Oxford Univ. Press.

[27] Alex Hanna, Emily Denton, Andrew Smart, and Jamila Smith-Loud. 2020. Towards a critical race methodology in algorithmic fairness. In Proceedings of the 2020 Conference on Fairness, Accountability, and Transparency. 501-512.

[28] Mortiz Hardt, Eric Price, and Nati Srebro. 2016. Equality of Opportunity in Supervised Learning. In Advances in Neural Information Processing Systems, Vol. 29.

[29] Anna Lauren Hoffmann. 2020. Rawls, Information Technology, and the Sociotech nical Bases of Self-Respect. In The Oxford Handbook of Philosophy of Technology.

[30] Simon Jackman. 2008. Measurement. In The Oxford Handbook of Political Methodology. Oxford Univ. Press, Chapter 9.

[31] Matthew Joseph, Michael Kearns, Jamie Morgenstern, Seth Neel, and Aaron Roth. 2017. Fair algorithms for infinite and contextual bandits. FATML, preprint arXiv:1610.09559 (2017)

[32] Matthew Joseph, Michael Kearns, Jamie H Morgenstern, and Aaron Roth. 2016. Fairness in learning: Classic and contextual bandits. NIPS (2016).

[33] Os Keyes. 2018. The misgendering machines: Trans/HCI implications of automatic gender recognition. Proceedings of the ACM on Human-Computer Interaction 2, CSCW (2018), 1-22.

[34] Jon Kleinberg, Sendhil Mullainathan, and Manish Raghavan. 2017. Inherent trade-offs in the fair determination of risk scores. ITCS (2017).

[35] Brian N Larson. 2017. Gender as a variable in natural-language processing: Ethical considerations. In Proc. of First Workshop on Ethics in Natural Language
Processing.

[36] Jane Loevinger. 1957. Objective tests as instruments of psychological theory. Psychological Reports 3, 3 (1957), 635-694.

[37] Kristian Lum and William Isaac. 2016. To predict and serve? Significance 13, 5 (2016), 14-19.

[38] Donald Martin, Jr, Vinodkumar Prabhakaran, Jill Kuhlberg, Andrew Smart, and William S Isaac. 2020. Extending the Machine Learning Abstraction Boundary: A Complex Systems Approach to Incorporate Societal Context. arXiv preprint arXiv:2006.09663 (2020).

[39] Sandra G Mayson. 2018. Bias in, bias out. Yale Law fournal 128 (2018), 2218.

[40] Samuel Messick. 1987. Validity. In ETS Research Report Series.

[41] Sendhil Mullainathan and Jann Spiess. 2017. Machine learning: an applied econometric approach. Journal of Economic Perspectives 31, 2 (2017), 87-106.

[42] Deirdre K Mulligan, Colin Koopman, and Nick Doty. 2016. Privacy is an essentially contested concept: a multi-dimensional analytic for mapping privacy. Phil. Trans. R. Soc. A 374, 2083 (2016), 20160118.

[43] Ziad Obermeyer, Brian Powers, Christine Vogeli, and Sendhil Mullainathan. 2019. Dissecting racial bias in an algorithm used to manage the health of populations. Science 366, 6464 (2019), 447-453.

[44] Alexandra Olteanu, Carlos Castillo, Fernando Diaz, and Emre Kiciman. 2019. Social data: Biases, methodological pitfalls, and ethical boundaries. Frontiers in Big Data 2 (2019), 13.

[45] National Committee on Vital and Health Statistics (NCVHS). 2012. Re: Development of Standards for the Collection of Socioeconomic Status in Health Surveys Conducted by the Department of Health and Human Services. https: //www.ncvhs.hhs.gov/wp-content/uploads/2014/05/120622lt.pdf

[46] Cathy O'Neil. 2016. Weapons of Math Destruction. Broadway Books.

[47] Samir Passi and Solon Barocas. 2019. Problem Formulation and Fairness. In Proceedings of the Conference on Fairness, Accountability, and Transparency (FAT '19). ACM, New York, NY, USA, 39-48. https://doi.org/10.1145/3287560.3287567

[48] Geoff Pleiss, Manish Raghavan, Felix Wu, Jon Kleinberg, and Kilian Q Weinberger. 2017. On fairness and calibration. NIPS (2017).

[49] Kevin M Quinn, Burt L Monroe, Michael Colaresi, Michael H Crespin, and Dragomir R Radev. 2010. How to analyze political attention with minimal assumptions and costs. American fournal of Political Science 54, 1 (2010), 209-228.

[50] Anna Roberts. 2018. Arrests as guilt. Ala. L. Rev. 70 (2018), 987.

[51] SAS. [n. d.]. EVAAS for K-12 Statistical Models. Available at https://www.sas.com/content/dam/SAS/en_us/doc/whitepaper1/sas-evaask12-statistical-models-107411.pdf, accessed 08-23-2018.

[52] Andrew D Selbst, Sorelle Friedler, Suresh Venkatasubramanian, Janet Vertesi, et al. 2019. Fairness and Abstraction in Sociotechnical Systems. In ACM Conference on Fairness, Accountability, and Transparency $\left(F A T^{*}\right)$.

[53] Stephen G Sireci. 1998. The construct of content validity. Social Indicators Research 45, 1-3 (1998), 83-117.

[54] Catalina L Toma, Jeffrey T Hancock, and Nicole B Ellison. 2008. Separating fact from fiction: An examination of deceptive self-presentation in online dating profiles. Personality and Social Psychology Bulletin 34, 8 (2008), 1023-1036.

[55] Human Rights Watch. 2020. UK: Automated Benefits System Failing People in Need. https://www.hrw.org/news/2020/09/29/uk-automated-benefits-systemfailing-people-need 